\title{
Evolution of Silicon Parameters due to Irradiation at the LHC
}

\section{Frank Hartmann* for the ATLAS, CMS Tracker and LHCb VELO collaboration}

Karlsruhe Institute of Technology, Karlsruhe, Germany

E-mail: Frank.Hartmann@cern. ch

The silicon tracking and vertexing detectors at the LHC are built to sustain fluxes of $10^{14}$ or even $10^{15} 1 \mathrm{MeV} / \mathrm{cm}^{2}$ _neutron_equivalent particles depending on their radial position. According to the original design the outer layers should last up to integrated luminosities of $\mathscr{L}=500 \mathrm{fb}^{-1}$ or even more while the inner ones are, in some cases, planned to be exchanged after some years. Simulations had been done to model radiation levels at the different positions in space of the detectors with respect to the delivered luminosity while radiation damage evolution of the sensors has been modelled and empirically determined. It is crucial to measure and understand the radiation damage evolution and compare it with the original model to predict the lifetime of the detectors and give first insights to the design of the next generation of radiation tolerant sensors. This contribution describes the strategies of the different LHC detector groups: ATLAS, CMS and LHCb VELO to measure the evolution of the critical parameters and how they compare with the available models. It also provides the first glimpses of the results at early stages below and around integrated luminosities of $\mathscr{L}=40 \mathrm{pb}^{-1}$ to $1 \mathrm{fb}^{-1}$. The goal of this document is to present the different techniques and to start a common discussion between the experiments of the next steps.

The 20th Anniversary International Workshop on Vertex Detectors - VERTEX 2011

June 19 - 24, 2011

Rust, Lake Neusiedl, Austria

\footnotetext{
* Speaker.
} 


\section{Radiation Damage and Different Strategies to Measure Basic Parameters}

The currently most frequently used n-type FZ sensor material at the LHC is very well understood with respect to bulk damage caused by radiation $\left(\Phi_{e q}\right)$ and the subsequent temperature dependant time evolution (annealing) and is extensively described in [1, 2, 3]. For n-FZ material, the fluence of different particles and different energies can be normalized via "non ionizing energy loss (NIEL)" calculations to $1 \mathrm{MeV}$ neutron equivalent $\left(1 \mathrm{MeV}_{e q}\right)$; damage from proton and neutron irradiation sums up.

The main two sensor parameters changing with irradiation are the reverse bias currents and the depletion voltage; while the currents can be measured directly and almost continuously the determination of depletion voltages needs special dedicated measurements. In addition both parameters behave differently with respect to annealing and temperature.

ATLAS [4] and LHCb VELO [5] at the LHC[6] operate below zero degree Celsius stopping the annealing process almost completely and these experiments only need to take account of the annealing during shutdowns or controlled warm-up periods. CMS [7], due to some cooling and sealing problems, operates still above zero degree Celsius and therefore experiences constant annealing.

ATLAS SCT has a very impressive resolution on the current measurement on their power supply channels at the $10 \mathrm{nA}$ level, while CMS SST (larger number of modules per HV channel), CMS pixel and LHCb VELO accuracy lies more in the $0.2-1 \mu \mathrm{A}$ range. In addition CMS strips and ATLAS pixel instrumented a small number of boards with high precision readout (10-50 nA). In addition ATLAS can measure the current of individual pixels at a resolution of $125 \mathrm{pA}$ while CMS measures dark currents on module level via an ADC circuit on the hybrid with an accuracy of $\sim 1 \mu \mathrm{A}$. All currents are basically measured continuously. In addition LHCb VELO conducts weekly current vs. voltage scans as a sanity check of the system. First results of current evolution are shown in section 2 .

The strategies devised to determine the depletion voltage vary between the detector groups. ATLAS pixel group measures the crosstalk between pixels by injecting test charges into single pixels for several voltages. With n-in-n pixel sensors, where the depletion zone builds up from the back and reaches the pixel side at the depletion voltage this can be done pre-Space Charge Type Inversion and outside stable beams. ATLAS SCT group measures the efficiency and depletion depth outside beam periods vs. voltage. CMS pixel, CMS SST and the LHCb VELO uses dedicated beam time to measure signal and efficiency versus voltage. CMS SCT and LHCb VELO also do noise vs. voltage studies outside beam periods as complementary measurements. First results and more details about depletion voltage studies are presented in section 3.

With the actual initial low luminosity (and thus low radiation damage) the results are more qualitative in nature than quantitative and serve as first anchor point and should be used to establish good and defined procedures. They also serve as first ingredients to improve the simulations.

\section{Results on Leakage Current Changes and Comparison with FLUKA prediction}

Radiation levels at the different parts of the detectors have been previously simulated by tools like FLUKA[8], taking into account both primary interaction and interactions with the detector, support structure and services. Earlier studies supplied numbers for charged and neutral particles 
separately but recent versions calculated directly $1 \mathrm{MeV}_{e q}$ numbers with fine granularity of position. The goal today is now to fold actual measurement values and simulation predictions, to understand the measurements itself but also to improve the simulation tools. The reverse bias currents, which can be easily measured almost continuously are well suited for the comparison between simulation and $1 \mathrm{MeV}_{e q}$ fluences. Also the instantaneous currents driven by the continuous radiation of charged particles can be compared to the prediction. In addition the local occupancy values derived from data taking can be used to check the simulated prediction.

Reverse bias current increases with fluence $\Delta I \sim \alpha(T, t) \Phi_{e q}$ where $\Phi_{e q}$. is the equivalent particle fluence, $\alpha$ the damage factor, $\mathrm{T}$ the temperature and $\mathrm{t}$ is time; then the current decreases for all silicon materials with time. The exponential annealing decay term depends strongly on temperature. Operation below zero almost freezes the annealing and at the same time limits the currents, while high temperature operation increase currents but also accelerates current annealing (decrease). CMS operates still above zero degree Celsius, therefore experiences high currents, the current decrease due to active annealing on the other hand is enhanced. To understand the evolution of the reverse currents CMS has to take the annealing component in a timely manner into account. Fig. 1 displays the current evolution measured and simulated for one. Figure 2 presents
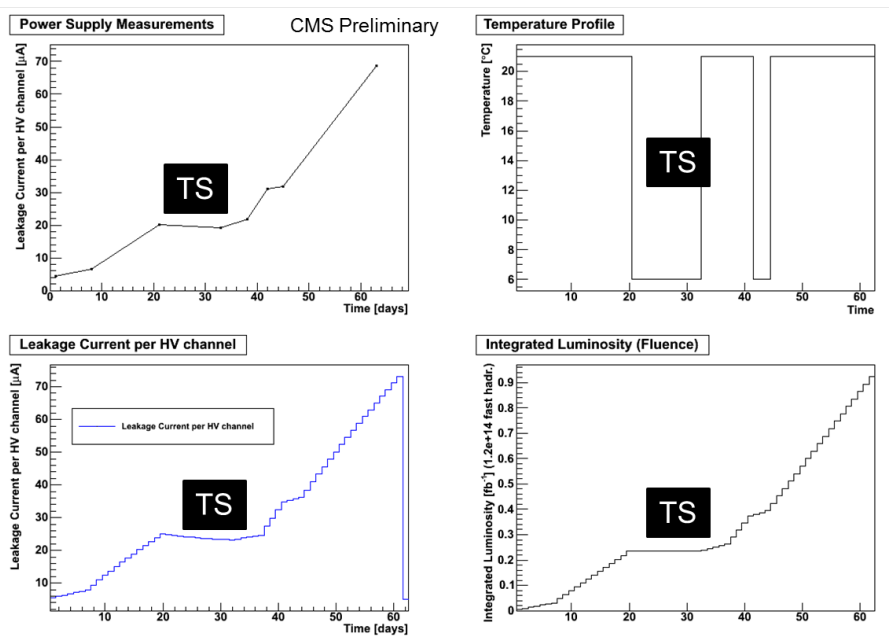

Figure 1: The left upper figure shows the reverse bias current measured by a dedicated high resolution power supply of CMS. The upper right presents a simplified profile of the silicon temperature during operation, LHC Machine Development and LHC short Technical Stop (TS). The lower right depicts the luminosity profile during the same period. The simulated current, taking the temperature and integrated luminosity into account, is shown in the lower left plot. The simulation algorithm works iteratively and calculates every 24 hours currents with the correct temperature based $\alpha$-factor. The simulation follows the real current in times of irradiation as well as in times without beam. With the elevated temperatures the annealing process is also active during the beam time. [9]

the evolution of reverse current for ATLAS, CMS and LHCb VELO. The linear increase with integrated luminosity is clearly visible. The annealing during the Extended Technical Stop 2010/11 is observed for ATLAS and LHCb VELO while the CMS plot starts in early 2011. Seeing the anticipated linear increase of current, the next question to be asked is, does it fit with the initial simulation? Does the absolute value fit? Do the radial and z-dependance fit? Figure 3 shows the 

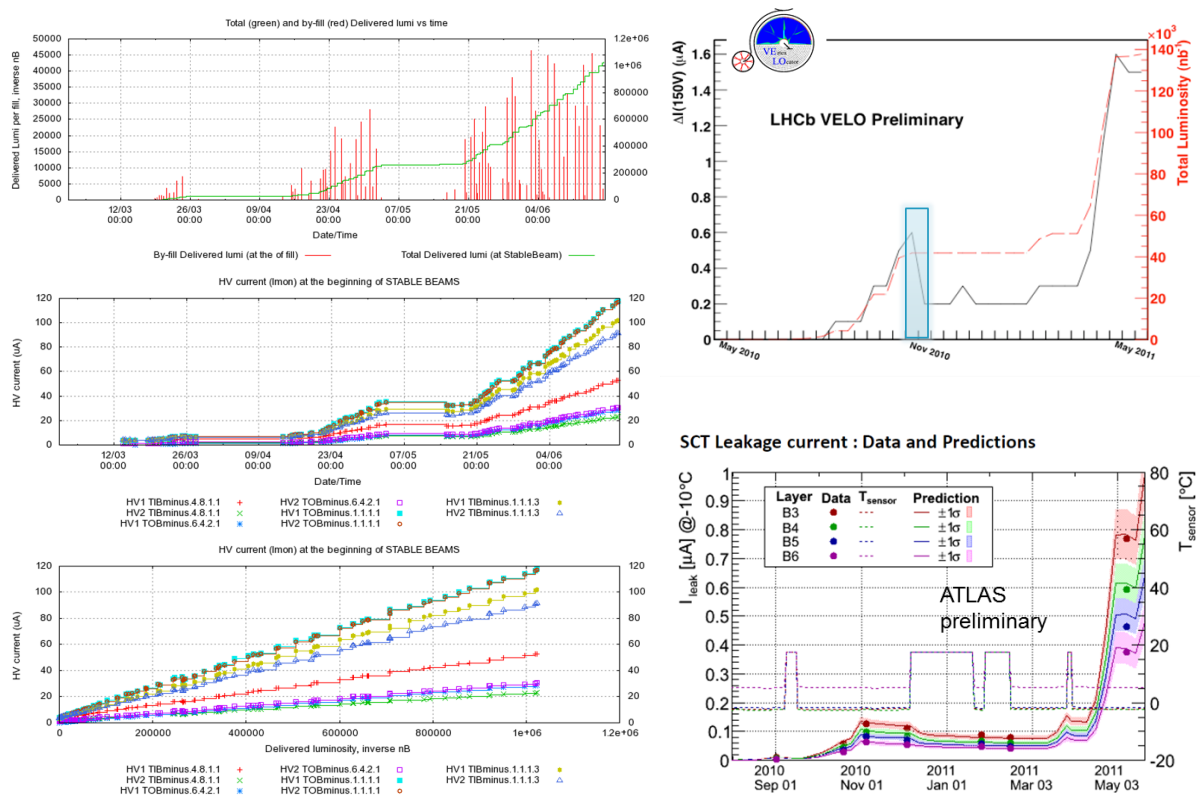

Figure 2: The linear increase with luminosity can be observed for all experiments. The three left plots presents the online web based tool output for HV currents for CMS. The upper one shows the instantaneous and integrated luminosity delivered by the LHC. To guarantee stable values, the first stored current value five minutes into Stable Beam is plotted for a certain number of different power channels. The different absolute current levels can be explained by the representation of modules at different radius (different $\phi$ ) with different thickness of sensors and also different temperatures. The lower plot depicts the linear $\Delta I \sim$ $\alpha(T, t) \cdot \Phi_{e q}$ curve. On the upper right the current evolution for LHCb VELO is shown. It follows the integrated luminosity. The decrease of current due to annealing during the Extended Technical Stop is clearly visible (LHCb stop began in November and did not participate in the Heavy Ion run 2010) [10]. The current evolution of the ATLAS sensors for the different barrel layers can be seen in the lower right plot including the simulated prediction. The temperatures during the different stops are also plotted and the decrease of current comes from annealing. [11]

simulated particle fluences in the ATLAS detector simulated by FLUKA and derived at some spots from the measured leakage currents. As we see in Fig. 2 the situation at CMS is not trivial because the different temperatures for the different modules and HV channels serve a different number of sensors and of different sensor sizes/volumes too. After normalizing everything to $\mathrm{T}=20^{\circ} \mathrm{C}$ and standard volume of $1 \mathrm{~cm}^{3}$ the leakage currents and their increase with radiation follow a distinct radial dependence $I \sim r^{-b}$. The power law does not follow the original simulation stated in the CMS TDR. The match of the most recent FLUKA simulation taking the real geometry and having values scored to $\left[\frac{\mu A}{f b^{-1} \mathrm{~cm}^{3}}\right]$ are presented in Fig. 4 and Fig. 5 shows the situation for the full range in $r$ and $z$ of the CMS strip Tracker.

In general, initial measurements confirm the modelling of evolution of sensor currents with increasing radiation - increasing with fluence but also decreasing with annealing. The measurement match the simulated expectation quite well, even with the current low radiation levels. An increase of the reverse currents increases power consumption and later noise. Operational limits are the maximum currents from the power supply system, maximum cooling power of the system and in the extreme case thermal runaway. 


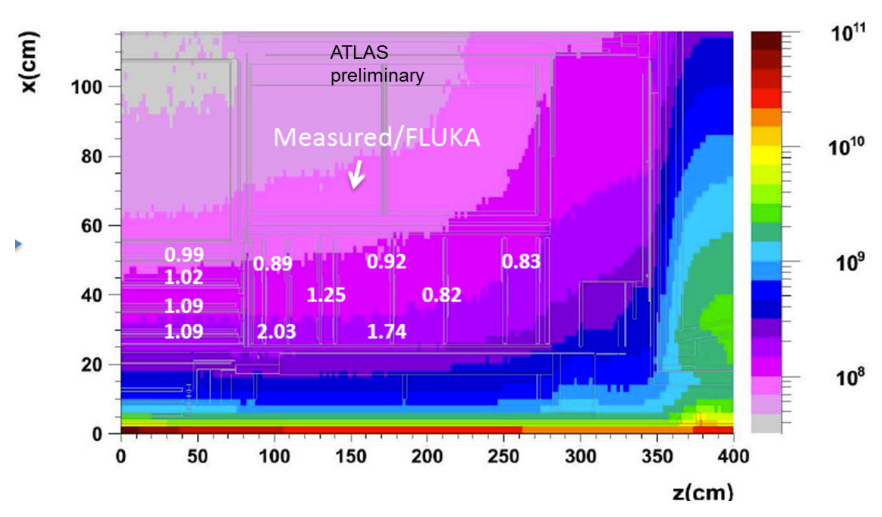

Figure 3: The color map shows the integrated fluences in $1 \mathrm{MeV}_{e q}$ particle numbers, obtained by a FLUKA simulation for ATLAS. These values were compared to the obtained actual fluences, derived from measured currents, temperature-normalized, averaged and multiplied the damage factor $\alpha$. The white numbers in the figure present the ratio of measured value to simulated FLUKA expectation. The match is very good (close to one) in the centre with some discrepancies in the inner end cap region. [11]
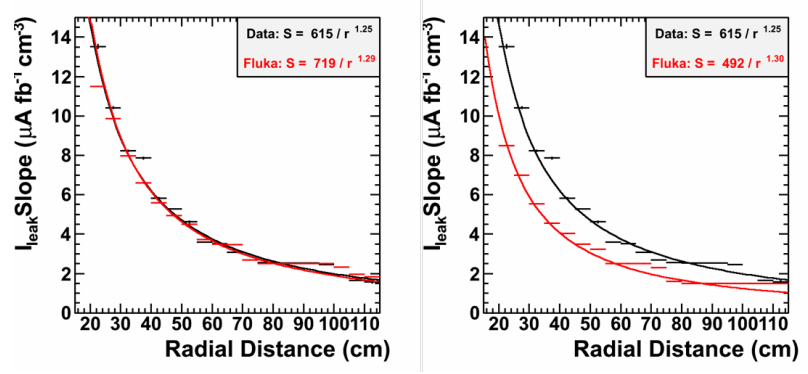

Figure 4: The plots show the comparison of $\left[\frac{\mu A}{\mathrm{fb}^{-1} \mathrm{~cm}^{3}}\right]$ between FLUKA simulation (red curve) and measured data (black curve). The FLUKA simulation output gives the fluence in the CMS Tracker per single pp collision at $14 \mathrm{TeV}$ expressed in $1 \mathrm{MeV}_{\text {eq }}$. The difference between the left and the right plot is the uncertainty on how to convert from a $14 \mathrm{TeV}$ simulation to the real situation of $7 \mathrm{TeV}$ collisions. In the left plot only the cross section was corrected to $77 \mathrm{mb}$, while in the right one also the theoretical multiplicity correction of $\sqrt{7 / 14}$ was included. The $\alpha$ factor to convert from fluence (FLUKA output) to current was assumed to be $4.85 \cdot 10^{-17} \mathrm{~A} / \mathrm{cm}$ corresponding to $20^{\circ} \mathrm{C}$ and $\sim 5$ days of annealing. The radial dependence matches very well $\left(I \sim r^{-1.25}\right)$ while the offset is still a bit uncertain. Also the continuous annealing changes the slopes $\left[\frac{\mu A}{f b^{-1} \mathrm{~cm}^{3}}\right]$ vs. time and a different $\alpha$ has to be used at a later time. [9]

\subsection{Dedicated Radiation Monitoring Devices in ATLAS}

ATLAS has instrumented specific locations with dedicated radiation monitoring instrumentation to determine the dose values using radiation sensitive p-MOS transitors (RADFETs) and calibrated diodes. The idea is to have independent measurements to determine the fluence (charged and neutral particles) and dose (charged particles) at these locations and to extrapolate to other regions using the simulations. Figure 6 shows the measurements and simulated prediction for dose and fluence. 


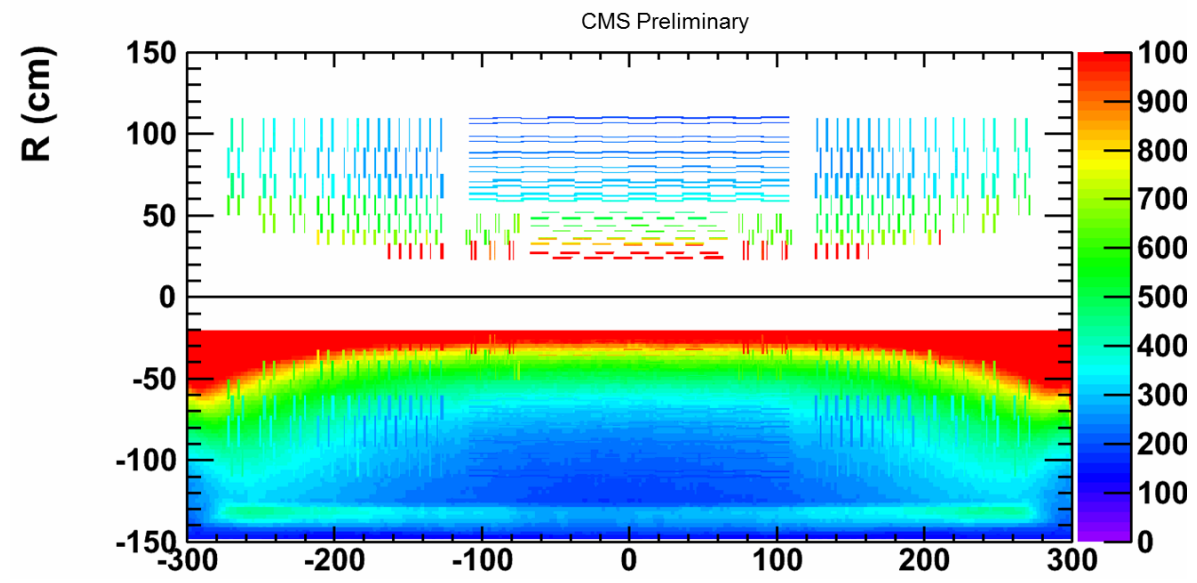

Figure 5: Comparison of measured and normalized current slopes $\left[\frac{\mu A}{f b^{-1} \mathrm{~cm}^{3}}\right]$ with anticipated simulated ones. The upper part of the figure displays the current slopes for the different HV channels. The lower parts presents the slopes superimposed on the derived ones from simulation. At locations where the modules are "invisible" the simulation fits perfectly to the measured value. The HV channels in the end cap region serve several modules spanning a large radius thus the averaged channels cannot perfectly match. [9]
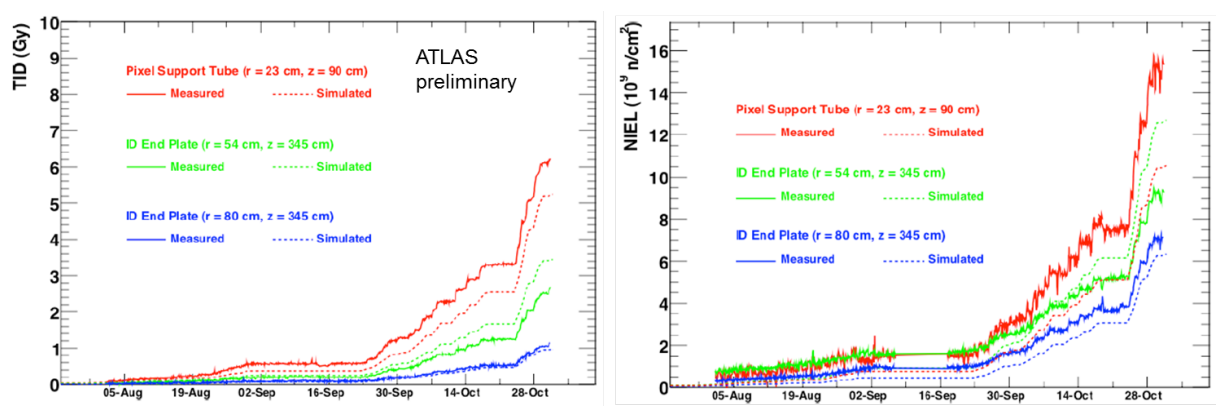

Figure 6: ATLAS Radmon detectors. At ATLAS, dedicated radiation sensitive p-MOS transitors (RADFETs) measure the localized doses. They are displayed in the left figure. The right plot shows the particle fluence measured by calibrated diodes (current measured). Both plots superimpose the expected values from simulation. [11]

\section{Results on Depletion Voltage Changes}

The $V_{\text {dep }}$ is proportional to $\left|N_{e f f}\right|$ while $N_{e f f}$ changes with $\Phi_{e q}$. For n-FZ primarily the acceptors are created and thus the effective doping concentration drops first to intrinsic and then $\left|N_{e f f}\right|$ increases again ${ }^{1}$. Differently to the leakage current annealing, which only decreases the currents with time, the annealing of $\left|N_{e f f}\right|$ has two components with two different time constants, one beneficial component where defects "recombine" and a reverse one, arising from a reconfiguration of defects into clusters leading to the build up of additional deep levels in the energy band. The reverse component will increase the depletion voltage after SCSI and thus may drive it above the limiting operation voltage. The strategy for the LHC silicon detectors is to benefit from the beneficial part by controlled warm periods and otherwise keep the detectors below $0^{\circ} \mathrm{C}$ to freeze out the annealing

\footnotetext{
${ }^{1}$ Also called Space Charge Sign Inversion SCSI or simply inversion
} 
processes. For the CMS detector which does not yet operate "cold" the monitoring of development of the depletion voltage is mandatory ${ }^{2}$. But also with the current excellent LHC performance integrated luminosities might exceed the design value ${ }^{3}$ before new detectors can be deployed and so solid lifetime projections are necessary. The Hamburg Model [2] provides initial constants but we already learned from radiation studies that these should be adapted to the sensors in use which can be established by in-situ depletion voltage monitoring.

Furthermore depletion voltages cannot simply be measured continuously like currents and dedicated runs are needed to derive them. The most commonly used ones, established by the TEVATRON detectors [12] are the (1) noise vs. bias voltage scan or the (2) signal vs. voltage scan which happen during a collision run. The first method works well for n-in-n sensors (ATLAS, CMS pixel and LHCb VELO) before SCSI or for double sided sensors (CDF, D0); exhibiting a distinct kink in noise when the depletion zone (only above depletion voltage) reaches the segmented side. The LHCb VELO detector and the CMS SST ${ }^{4}$ exploits the noise vs. bias scan. CMS Pixel, CMS SST and LHCb VELO also make use of the signal vs. voltage scans. Fig. 7 shows the signal vs. voltage scans of CMS pixel, CMS SST and LHCb VELO.
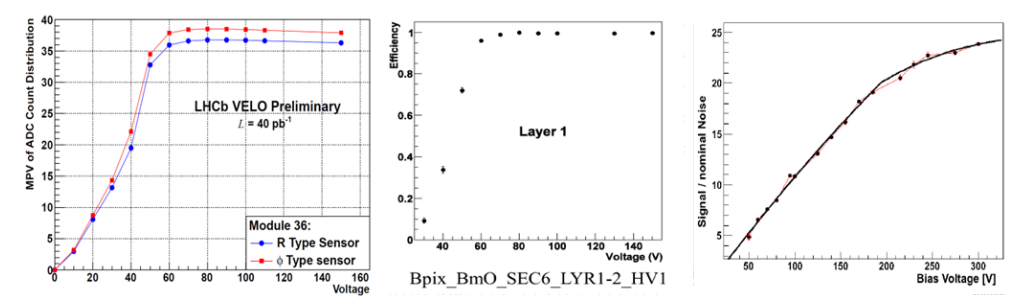

Figure 7: The charge collection efficiency for LHCb VELO[10], CMS pixel and CMS SST are shown. The distinctive kink at the depletion voltage can be easily identified for LHCb VELO and CMS pixel but not for the CMS SST operating in the nominal deconvolution mode (see later).

In the case of the VELO, the sensors are arranged perpendicular to the beam and therefore receives higher radiation damage at lower radii. The noise method is location sensitive thus different depletion voltages for different strip regions can be derived. Fig. 8 displays the expected radiation distribution versus radius and VELO stations as well as the first result after $\mathscr{L}=160 \mathrm{pb}^{-1}$.

The signal vs. noise or CCE (Charge Collection Efficiency) scan for LHCb VELO is fully automated and rotates through several defined patterns in around 2 hours. Every fifth sensor is designated as a test sensor. The others operate at nominal bias voltage and are used for particle tracking. The fitted particle tracks are extrapolated to the test sensors and the charge on the strips around the intercept point of the track is determined. Non-zero suppressed data is recorded to get the full charge down to zero charge even when operating under-depleted. The depletion voltage point derived from earlier lab capacitance vs. voltage scans matches the $80 \%$ signal plateau point which is now defined as in-situ depletion voltage. The results can be seen in Fig. 9.

\footnotetext{
${ }^{2}$ Initial simulations predict the penalty in depletion voltage by operating "'warm"' until the next Long Shutdown LS1 is only marginal.

${ }^{3}$ e.g. of $\mathscr{L}=500 \mathrm{fb}^{-1}$ for ATLAS and CMS

${ }^{4}$ The CMS chip APV is very sensitive to input capacitance $\sim$ backplane capacitance changes thus the noise vs. voltage method works here also for $\mathrm{p}$-in-n sensors. The noise is expected to minimise once the detector reaches its full depletion voltage and thus shows a plateau.
} 

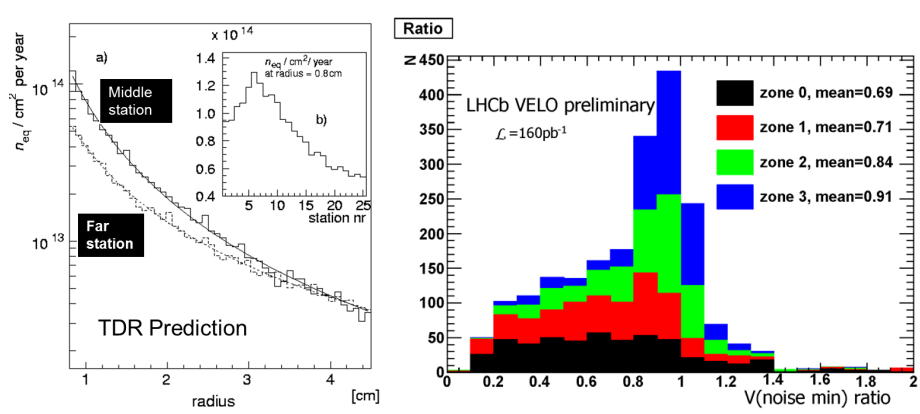

Figure 8: On the left, the LHCb VELO TDR prediction of particle fluences for the different radii and stations (position in $z$ ) can be seen, representing one year of operation corresponding to an integrated luminosity of $2 \mathrm{fb}^{-1}$ at the experiment. The figure on the right shows the extracted depletion voltage results normalized to the value measured at the start of the LHC physics operation. The distribution indeed shows that the depletion voltages of the innermost zone (black) of the sensors have changed most, and the outermost zone (blue) have changed least, in agreement with the expectation of a radial dependence of roughly $\sim r^{-1.9}$. The broad distributions can be explained by the different stations situated at different Z.[10]
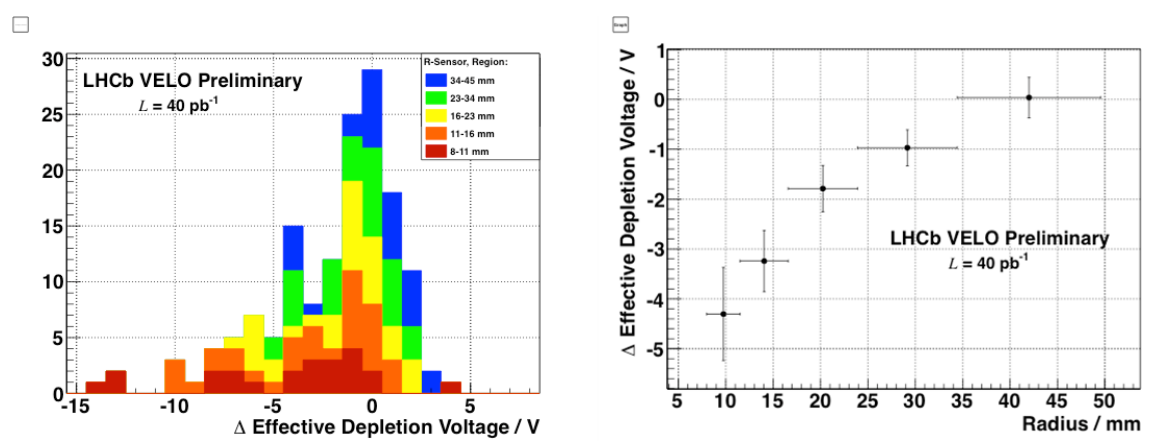

Figure 9: Development of the depletion voltage in the LHCb VELO. The plot shows the difference between initial depletion voltage and $\mathscr{L}=40 \mathrm{pb}^{-1}$. Due to the closeness to the beam inner regions of the sensors will invert even in the second year of operation, thus changes at even these low integrated luminosities are expected. The different radial regions are tagged by different colors while the broad distribution can be explained by the different detector stations. The mean difference values of the different regions are plotted on the right and follow roughly the expected $\sim r^{-1.9}$ power law. [5, 10]

CMS SST scans the full detector at once utilizing the pixel seeds to extrapolate tracks and only analyze on-track clusters for the fit. Monthly bias scans during collision for a subset (1-2\%) of the CMS pixel and SST detectors plus two to three full detector bias scans per year are planned. The CMS SST utilizes CMS Pixel seed tracks to extrapolate tracks and vice versa. Both partial and full scans have been conducted at the very first beams to obtain an anchor point for future measurements. Full automation of the scans is underway. The CMS SST method which is used to extract the depletion voltage from the noise vs. voltage and signal vs. voltage is described in detail in [13]. The method and first results of the signal vs. voltage scan of the full CMS SST are also presented here. With the APV chip operating in deconvolution mode ${ }^{5}$ the charge collection efficiency does

\footnotetext{
${ }^{5}$ This readout procedure uses a weighted sum of three sequentially stored output values. With this readout mode the effective time in which charge is collected is about $25 \mathrm{~ns}$, which is less than the transition time for under depleted sensors. More details can be found in Ref.[14].
} 
not show a very distinctive kink. The bias voltage has a crucial impact on the efficiency of collecting charge for silicon sensors. In order to quantify the effects CMS has modelled the change in charge collection efficiency based on three effects.

- The variation of the width of the depletion zone changes the amount of collected charge.

- The change in the drift velocity of charge carriers modifies the amount of charge collected during the integration time of the readout electronics.

- The change in the load capacitance has an influence on the shaping of the signal pulse and thus the collected charge.

Figure 10 demonstrates the different effects, shows the family of modelled curves and an example fit. Figure 11 demonstrates the quality of the method and compares the in-situ results for the SST
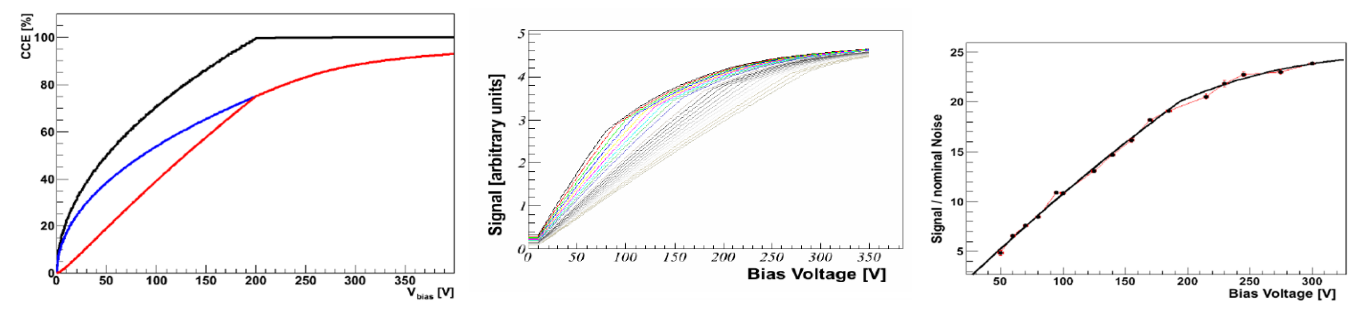

Figure 10: The first plot demonstrates the influence of the different effects on the charge collection efficiency - black represents the depletion width; blue the change of drift velocity and red the change of pulse shape due to change in load capacitance. The middle plot displays the family of modelled curves for the different depletion voltages, where each line represents a defined depletion voltage. The data curves are then compared to the modelled ones. The right plot shows an example of a real scan and its fit to the model curve. The method is very sensitive and the fit identifies the approximately same depletion voltage value as derived from a simple CV scan in the lab. [13]

inner barrel detector with results derived from CV measurements during the quality assurance or in several cases received from the producer.
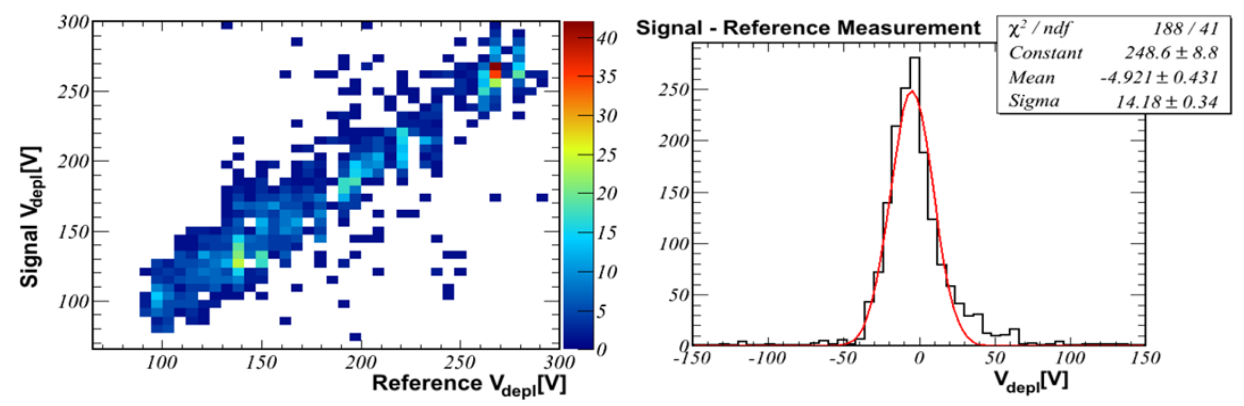

Figure 11: The correlation plots demonstrates the functionality of the fit method in comparison with the previous lab measurements. The scan was taken with one of the earliest LHC collision in 2009 beams where no radiation damage was expected and will serve as an anchor point for future scans for each individual module. [13] 


\section{Conclusion and Outlook}

The LHC silicon detector groups have developed their own strategies to monitor the evolution of silicon sensor parameters with the increasing integrated luminosity thus radiation damage. Leakage currents increase linearly with fluence as expected and first comparisons with expectation from simulation are encouraging. The methods to extract the depletion voltage in-situ have been successfully demonstrated by established signal or noise vs. voltage scans. In the VELO case the scans are already fully automated and first changes due to their closeness to the beam $(8 \mathrm{~mm})$ have been observed even for initial values of $\mathscr{L}=40 \mathrm{pb}^{-1}$. In the future higher levels of integrated luminosity will allow even better understanding. We believe the first steps are quite encouraging and allow us to test the values extracted against the Hamburg Model and allow projection into the future. They also allow us to predict the effect of warm up periods during the technical stops and may even support the upgrade planning.

\section{References}

[1] http://www.cern.ch/rd50

[2] M.Moll, Radiation Damage in Silicon Particle Detectors - Microscopic Defects and Macroscopic Properties, Ph.D.Thesis, DESY-THESIS-1999-040, December 1999, ISSN:1435-8085.

[3] Evolution of Silicon Sensor Technology in Particle Physics; Series: Springer Tracts in Modern Physics; http://www.springer.com/series/426, Vol. 231 (2009), ISBN: 978-3-540-25094-4

[4] The ATLAS Experiment at the CERN Large Hadron Collider. The ATLAS Collaboration. 2008, JINST 3 S08003.

[5] The LHCb Detector at the LHC. The LHCb collaboration. 2008, JINST 3 S0800

[6] LHC Machine. The LHC Collaboration. 2008, JINST 3 S08001.

[7] The CMS experiment at the CERN LHC. The CMS Collaboration. 2008, JINST 3 S08004.

[8] "The FLUKA code: Description and benchmarking" G. Battistoni, S. Muraro, P.R. Sala, F. Cerutti, A. Ferrari, S. Roesler, A. Fasso', J. Ranft, Proceedings of the Hadronic Shower Simulation Workshop 2006, M. Albrow, R. Raja eds., AIP Conference Proceeding 896, 31-49, (2007)

"FLUKA: a multi-particle transport code" A. Fasso, A. Ferrari, J. Ranft, and P.R. Sala, CERN-2005-10 (2005), INFN/TC_05/11, SLAC-R-773

[9] Private Communication CMS - radiation monitoring group

[10] Private Communication LHCb VELO - Chris Parkes

[11] Private Communication ATLAS - Stephen Mcmahon and Beniamino Di Girolamo

[12] J. Wagner et al., Operational experience with the silicon detector at CDF, NIMA 576 (2007) 220-222 M. Stanitzki for the CDF and DØ Collaborations, Radiation hardness experience with the CDF/DØ Silicon vertex detectors, NIMA 582 (2007) - VERTEX 2006

Miguel N. Mondragon et al., Operational experience with the CDF Run II Silicon Detector, NIMA 617 (2010)

[13] C. Barth et al., "'Evolution of silicon sensors characteristics of the current CMS tracker", Nucl.Instr.andMeth.A(2011), doi:10.1016/j.nima.2011.05.045

[14] M. Raymond,et al., The CMS Tracker APV25 0.25 mum CMOS Readout Chip, Sixth Workshop on Electronics for LHC Experiments, CERN/LHCC/2000-041,p.130. 